\title{
Metformin decreases high-fat diet-induced renal injury by regulating the expression of adipokines and the renal AMP-activated protein kinase/acetyl-CoA carboxylase pathway in mice
}

\author{
DAL KIM $^{1}$, JUNG EUN LEE ${ }^{1}$, YU JIN JUNG ${ }^{1}$, AE SIN LEE $^{1}$, SIK LEE $^{1}$, SUNG KWANG PARK $^{1}$, \\ SUHN HEE KIM ${ }^{2}$, BYUNG-HYUN PARK ${ }^{3}$, WON KIM ${ }^{1}$ and KYUNG PYO KANG ${ }^{1}$ \\ ${ }^{1}$ Department of Internal Medicine, Research Institute of Clinical Medicine and Diabetes Research Center, \\ ${ }^{2}$ Department of Physiology and Diabetes, ${ }^{3}$ Department of Biochemistry and Diabetes Research Center, \\ Chonbuk National University Medical School, Deokjin-gu, Jeonju 561-712, Republic of Korea
}

Received June 24, 2013; Accepted September 18, 2013

DOI: $10.3892 /$ ijmm.2013.1508

\begin{abstract}
Metabolic syndrome is characterized by insulin resistance, dyslipidemia and hypertension. These metabolic changes contribute to the development of obesity-induced kidney injury. AMP-activated protein kinase (AMPK) is a ubiquitous enzyme that is involved in the cellular metabolic response to metabolic stress. Metformin, an AMPK activator, has been reported to exert a protective effect against nonalcoholic steatohepatitis. However, little is known about its role in the pathogenesis of obesity-induced renal injury. The aim of this study was to investigate the effects of metformin on high-fat diet (HFD)-induced kidney injury. Obesity was induced by HFD ( $60 \%$ of total calories from fat, $20 \%$ protein and $20 \%$ carbohydrates) in 6-week-old C57BL/6 mice. Mice were fed HFD plus $0.5 \%$ metformin. The effects of metformin on HFD-induced renal injury were evaluated by determining metabolic parameters, serum adipokine levels and renal AMPK/acetyl-CoA carboxylase (ACC) activities, as well as a histological examination. HFD induced metabolic derange-
\end{abstract}

Correspondence to: Professor Kyung Pyo Kang, Department of Internal Medicine, Research Institute of Clinical Medicine and Diabetes Research Center, Chonbuk National University Medical School, 20 Geonjiro, Deokjin-gu, Jeonju 561-712, Republic of Korea E-mail:kpkang@chonbuk.ac.kr

Abbreviations: ACC, acetyl-CoA carboxylase; CRP, C-reactive protein; AMPK, AMP-activated protein kinase; HFD, high-fat diet; HOMA-IR, homeostasis model assessment of insulin resistance; IL-6, interleukin-6; MCP-1, monocyte chemoattactant protein-1; PAI-1, plasminogen activator inhibitor type 1 ; TGF- $\beta 1$, transforming growth factor- $\beta 1$; TNF- $\alpha$, tumor necrosis factor- $\alpha$; Vglom, glomerular volume

Key words: obesity, renal injury, insulin resistance, adipokine, AMP-activated protein kinase ment, systemic insulin resistance and glomerular mesangial matrix expansion. The administration of metformin reduced HFD-induced metabolic derangement and renal injury. The administration of metformin reduced the HFD-induced increase in adipokine expression and macrophage infiltration. Moreover, renal AMPK activity, which was decreased by HFD, was recovered following the administration of metformin; in addition, fatty acid oxidation was increased by the inhibition of ACC. These results indicate that metformin exerts beneficial effects on obesity-induced renal injury by regulating systemic inflammation, insulin resistance and the renal AMPK/ACC pathway. The clinical application of metformin to obese or early diabetic patients may be helpful in preventing obesity- or diabetes-related kidney disease.

\section{Introduction}

Although diabetes and hypertension are leading causes of chronic kidney disease worldwide, obesity is another health issue. Epidemiological studies have indicated that obesity and metabolic syndrome are risk factors for developing chronic kidney diseases (1-3). Insulin resistance is a well-known pathophysiological characteristic of obesity and it causes salt retention, sympathetic overactivity and endothelial dysfunction. These pathogenic factors lead to increased blood pressure, atherogenesis and microalbuminuria (4). Another hallmark of metabolic syndrome is a status of chronic inflammation, including elevated levels of pro-inflammatory markers, such as C-reactive protein (CRP), tumor necrosis factor- $\alpha$ (TNF- $\alpha$ ), interleukin-6 (IL-6) and plasminogen activator inhibitor type-1 (PAI-1) or reduced levels of anti-inflammatory molecules, such as adiponectin (5). These adipokines are produced and secreted by adipose tissue and are connected to obesity-related complications.

The pathophysiology of obesity-related renal injury remains obscure. Potential mechanisms of obesity-related renal injury include renal hemodynamic changes, increased insulin resistance, dyslipidemia, activation of the renin-angiotensin system, 
low-grade inflammation and oxidative stress (6). Among these, the modulation of insulin resistance and low-grade inflammation may be a potential therapeutic strategy for the prevention and treatment of obesity-related renal injury.

Metabolic stress, such as impaired adenosine-5'-triphosphate (ATP) production or accelerated ATP consumption activate the AMP-activated protein kinase (AMPK) system, which acts as a sensor of cellular energy metabolism (7). Among the pharmacological activators of AMPK, metformin is an oral hypoglycemic agent that is recommended as the first choice of treatment for patients with type 2 diabetes in the absence of contraindications (8). The cellular mechanisms of metformin involve the indirect activation of AMPK secondary to the inhibition of the mitochondrial respiratory chain complex $I$ in the regulation of hepatic glucose metabolism (9-11). In addition to these metabolic effects, AMPK activation may exert beneficial effects by reducing systemic inflammation and improving insulin resistance in diabetic or obese patients (12). Another beneficial effect of metformin is that it improves hepatic lipid metabolism. AMPK activation by metformin regulates acetyl-CoA carboxylase (ACC) activity by its phosphorylation $(13,14)$. ACC is a rate-controlling enzyme for the synthesis of malonyl-CoA, which plays a critical role in the synthesis of fatty acids and the inhibition of mitochondrial fatty acid oxidation in the liver $(14,15)$. Therefore, modulation of the renal AMPK/ACC pathway may improve HFD-induced renal lipotoxicity. Decleves et al (16) demonstrated that AMPK mediates the early renal effects of a high-fat diet (HFD) and showed that the activation of AMPK by treatment with 5-aminoimidazole4-carboxamide-1- $\beta$-D-ribonucleoside (AICAR) for 1 week induced protective effects against HFD-related kidney disease. Metformin, as an AMPK activator, has been reported to exert a protective effect against non-alcoholic steatohepatitis (17). To date, however, only a few studies have examined its effects on HFD-induced renal injury.

In this context, we conducted this study to examine the effects of metformin on HFD-induced renal injury and to clarify the relevant mechanisms. Our results demonstrate that metformin exerts beneficial effects on obesity-related renal injury by regulating the systemic expression of adipokines, insulin resistance and the renal AMPK/ACC pathway.

\section{Materials and methods}

Animal experiments. The animal experimental protocol was reviewed and approved by the Institutional Animal Care and Use Committee of Chonbuk National University, Jeonju, Korea (CBU 2012-0015). Male C57BL/6 mice (6 weeks old, weighing 17-19 g) were purchased from Orient Bio Inc. (Seoul, Korea) and maintained in a room under controlled temperature $\left(23 \pm 1^{\circ} \mathrm{C}\right)$, humidity and lighting (12-h light/12-h dark cycle) conditions and allowed free access to water. The mice were divided into 3 experimental groups: i) a group fed a standard diet (control; Con, $n=12)$ in which the mice were fed standard chow (10\% of kcal from fat); ii) a group fed HFD $(n=12)$ in which the mice were fed HFD (60\% of total calories from fat, $20 \%$ protein and $20 \%$ carbohydrates) from Research Diets (New Brunswick, NJ, USA); and iii) a group fed HFD plus metformin (HFD + Met, $\mathrm{n}=12$ ) in which the mice were fed HFD mixed with $0.5 \%$ (w/w) metformin (Sigma Chemical Co., St. Louis, MO, USA) for 12 weeks. During the 12 weeks of the experimental period, body weight and food intake were measured every 2 weeks. At the end of the experiment, the mice were anesthetized using ketamin $(100 \mathrm{mg} / \mathrm{kg})$ and xylazine $(10 \mathrm{mg} / \mathrm{kg})$ after a 6 -h fast and then blood was collected by intracardiac puncture. The kidneys were harvested and their weight was measured.

Measurement of metabolic parameters, adipokines and transforming growth factor (TGF)- $\beta 1$. At the end of the experiment, the mice were placed in metabolic cages to collect $24 \mathrm{~h}$ urine samples for the measurement of urine albumin and creatinine excretion. Urinary albumin excretion was measured using the Albuwell M kit (Exocell, Philadelphia, PA, USA). Urinary creatinine was measured using the Creatinine Companion (Exocell). Urinary albumin excretion was expressed as the ratio of urinary albumin to creatinine. For blood chemistry, serum aspartate aminotransferase (AST) and alanine aminotransferase (ALT) levels were measured by enzymatic methods (Neodin Laboratories, Seoul, Korea). Serum glucose, total cholesterol, triglyceride and free fatty acid levels were determined by enzymatic methods (Wako Diagnostics, Richmond, VA, USA). Serum insulin, TNF- $\alpha$, IL-6, leptin, resistin, total PAI-1 and monocyte chemoattactant protein-1 (MCP-1) levels were measured using a mouse serum adipokine milliplex kit (Millipore, Billerica, MA, USA). Serum adiponectin (Millipore) and renal TGF- $\beta 1$ (R\&D Systems, Minneapolis, MN, USA) levels were measured by enzymelinked immunosorbent assay (ELISA) according to the manufacturer's instructions. The homeostasis model assessment of insulin resistance (HOMA-IR) was calculated using the following equation: fasting glucose $(\mathrm{mmol} / \mathrm{l}) \mathrm{x}$ fasting insulin $(\mathrm{mU} / 1) / 22.5$. The intraperitoneal glucose tolerance test was performed as follows: briefly, the mice were fasted for $6 \mathrm{~h}$ and a fasting glucose level was obtained from venous blood from a tail vein clip. Glucose ( $1 \mathrm{mg} / \mathrm{g}$ body weight) was injected intraperitoneally and blood glucose levels were measured at 30,60 and $120 \mathrm{~min}$.

Histological examination. The kidneys were fixed in $4 \%$ paraformaldehyde and embedded in paraffin. The block was cut into 5- $\mu \mathrm{m}$ sections and stained with periodic acid-Schiff (PAS). All the slides were evaluated by 2 observers who were blinded to the origins of the slides using a Zeiss Z1 microscope (Carl Zeiss, Göttingen, Germany). The extent of the mesangial area was measured by assessing the PAS-positive and nuclei-free area in the mesangium. The glomerular area was determined along the outline of the capillary loop. The glomerular volume (Vglom) was estimated according to the Weibel and Gomez method as follows: Vglom = mean glomerular $\operatorname{area}^{1.5} \times 1.38 / 1.01$, where 1.38 is $\beta$, the shape coefficient of the sphere, and 1.01 is the size distribution coefficient of glomeruli assuming a $10 \%$ coefficient of variation of diameter, as previously described (18).

Transmission electron microscopy was performed as previously described (19). The kidneys were fixed in $0.1 \mathrm{M}$ cacodylate buffer with $2.5 \%$ glutaraldehyde. Ultrastructural changes in renal tissue, particularly extent of the effacement of podocyte foot process and tubular changes were examined using a Hitachi H-7600S transmission electron microscope (Hitachi, Tokyo, Japan). 
Table I. Biochemical parameters in obese mice following treatment with metformin.

\begin{tabular}{lcrr}
\hline Parameter & \multicolumn{1}{c}{ Con } & HFD & HFD + Met \\
\hline Body weight (g) & $29.5 \pm 0.72$ & $50.4 \pm 2.62^{\mathrm{a}}$ & $38.8 \pm 3.21^{\mathrm{b}}$ \\
Kidney weight (g) & $0.18 \pm 0.02$ & $0.21 \pm 0.01^{\mathrm{a}}$ & $0.18 \pm 0.02^{\mathrm{b}}$ \\
Glucose (mg/dl) & $119.3 \pm 18.7$ & $218.3 \pm 11.3^{\mathrm{a}}$ & $131.25 \pm 22.2^{\mathrm{b}}$ \\
AST (IU/l) & $73.5 \pm 15.3$ & $215.5 \pm 46.6^{\mathrm{a}}$ & $160.8 \pm 53.3^{\mathrm{b}}$ \\
ALT (IU/l) & $36.3 \pm 7.2$ & $246.3 \pm 44.3^{\mathrm{a}}$ & $92.3 \pm 53.9^{\mathrm{b}}$ \\
Cholesterol (mg/dl) & $73.3 \pm 5.3$ & $154 \pm 15.9^{\mathrm{a}}$ & $129 \pm 9^{\mathrm{b}}$ \\
Triglycerides (mg/dl) & $138.3 \pm 12.6$ & $159.2 \pm 20.2^{\mathrm{a}}$ & $154.2 \pm 14.7$ \\
Free fatty acids (mmol/l) & $1.78 \pm 0.23$ & $1.84 \pm 0.32$ & $1.8 \pm 0.31$ \\
\hline
\end{tabular}

${ }^{\mathrm{a}} \mathrm{P}<0.05$ vs. (Con); ${ }^{\mathrm{b}} \mathrm{P}<0.05$ vs. HFD-fed group. Con, control group fed standard diet; HFD, group fed high-fat diet; HFD + Met, group fed high-fat diet in conjunction with metformin; AST, aspartate aminotransferase; ALT, alanine aminotransferase.

Immunofluorescence staining was performed as previously described (20). For immunofluorescence staining, freshly frozen renal tissues were fixed with $4 \%$ paraformaldehyde, permeabilized in $1 \%$ Triton X-100 and then incubated with blocking buffer. Subsequently, the samples were incubated with rabbit anti-type I and type IV collagen (Cosmo Bio Co., Ltd., Tokyo, Japan), rabbit anti-mouse fibronectin (BD Biosciences, Franklin Lakes, NJ, USA), rat anti-mouse synaptopodin (Acris Antibodies, Herford, Germany) and rat anti-mouse F4/80 (Serotec, Oxford, UK) antibodies. The slides were exposed to Cy3-labeled secondary antibody (Chemicon, Temecula, CA, USA). Nuclear staining was performed using DAPI. Digital images were obtained using a Zeiss LSM 510 confocal microscope (Carl Zeiss). The area fraction (\%) of fibronectin, type I collagen, type IV collagen and synaptopodin in each glomerulus was measured at a magnification of $\mathrm{x} 400$. For morphometric analysis, a total of 30 glomeruli cut at their vascular poles in each kidney were examined using ImageJ software (http://rsb.info.nih.gov/ij).

Western blot analysis. Western blot analsyis was performed as previously described (21). The kidney tissues were homogenized in phosphate-buffered saline (PBS) with protease inhibitor cocktail (Calbiochem, San Diego, CA, USA) and the protein concentration was quantified by the Bradford protein assay. The samples (30 $\mu \mathrm{g}$ protein/lane) were mixed with sample buffer, boiled for $6 \mathrm{~min}$, separated by SDS-polyacrylamide (8-15\%) gel electrophoresis and then electroblotted onto nitrocellulose membranes (Bio-Rad Laboratories, Hercules, CA, USA). The membranes were then blocked with $5 \%$ non-fat dry milk in Tris-buffered saline

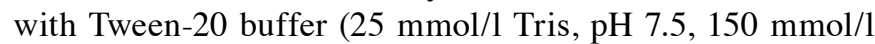
$\mathrm{NaCl}$, and $0.1 \%$ Tween-20) for $1 \mathrm{~h}$ and then incubated overnight at $4^{\circ} \mathrm{C}$ with rabbit anti-phospho-AMPK $\alpha$ (Thr172) and rabbit anti-phospho-ACC (Ser79) antibodies (Cell Signaling Technology, Danvers, MA, USA). The blots were washed with Tris-buffered saline with Tween-20 buffer and incubated with horseradish peroxidase-conjugated anti-rabbit IgG. Signals were visualized using a chemiluminescent detection kit according to the manufacturer's instructions (Amersham
Pharmacia Biotech, London, UK). The membranes were then re-probed with rabbit anti-AMPK $\alpha$ and ACC antibodies (Cell Signaling Technology) to verify equal loadings of protein in each lane. All signals were analyzed by densitometric scanning (LAS-3000; Fuji Film, Tokyo, Japan).

Statistical analysis. Data are expressed as the means \pm SD. Multiple comparisons were examined for significant differences using ANOVA, followed by individual comparison with the Tukey post hoc test; a value of $\mathrm{P}<0.05$ was considered to indicate a statistically significant difference.

\section{Results}

Metformin improves HFD-induced systemic metabolic derangement. At the end of the 12-week experimental period, the physical and biochemical profiles in each group were calculated (Table I). The group fed HFD showed a significant increase in body and kidney weight compared with the control group (fed standard diet). The HFD-fed mice showed significantly high levels of AST/ALT, glucose, cholesterol and triglyceride compared with the control mice. The levels of the metabolic parameters, apart from triglyceride levels, were significantly lower in the mice administered metformin compared with the HFD-fed mice. In addition, there were no significant differences in the serum levels of free fatty acids among the experimental groups.

Since obesity is associated with insulin resistance, we conducted an intraperitoneal glucose tolerance test at the end of the experimental period. The HFD-fed mice showed glucose intolerance; however, the administration of metformin significantly attenuated the HFD-induced glucose intolerance (Fig. 1A). Serum insulin levels significantly increased in the HFD-fed group compared with the control group; however, metformin suppressed the HFD-induced increase in insulin levels. In addition, the HFD-fed group showed insulin resistance by HOMA-IR; however, metformin attenuated the HFD-induced increase in insulin resistance (Fig. 1B and C). To evaluate renal injury, we measured urinary albumin excretion at the end of the experiment. Urinary albumin excretion 

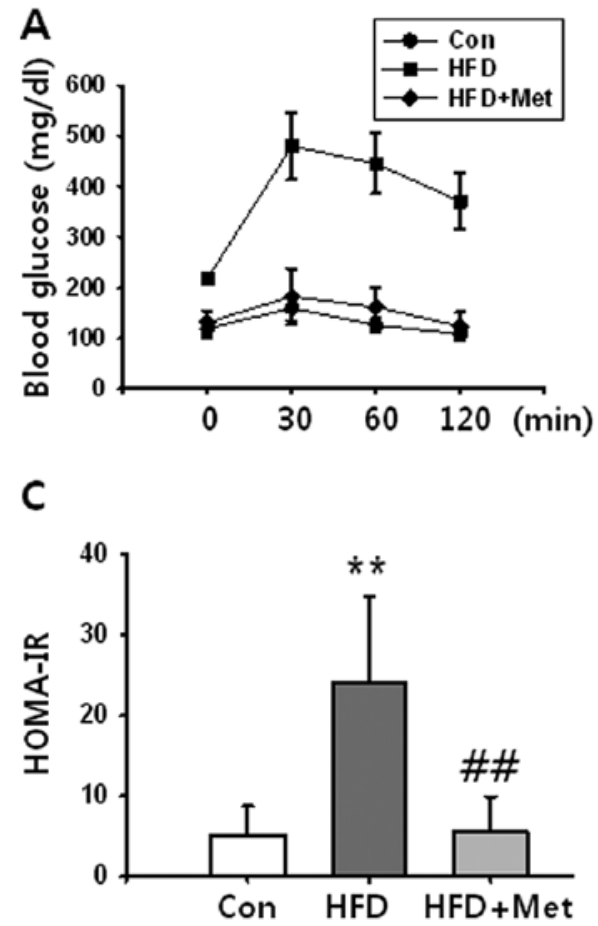

B

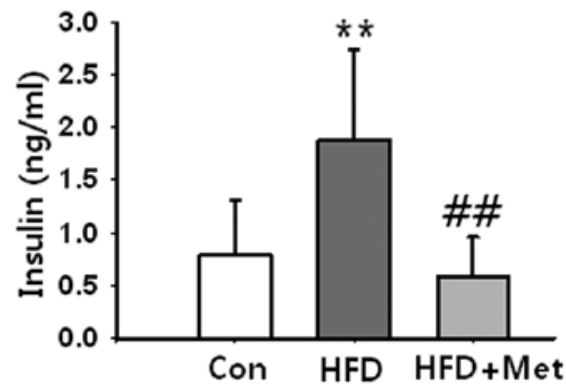

D

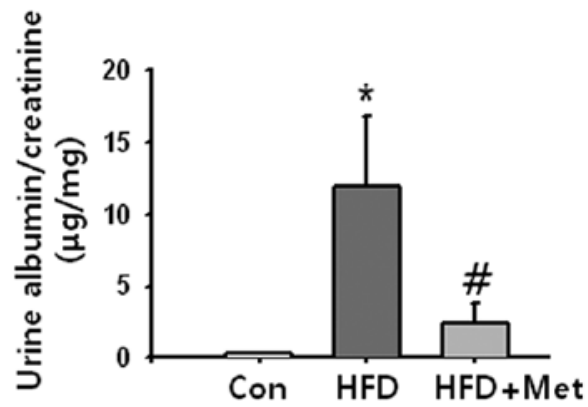

Figure 1. Effects of metformin on HFD-induced glucose intolerance and insulin resistance. (A) Intraperitoneal glucose tolerance test at the end of the experimental period ( $\mathrm{n}=12$ mice/group). (B) Effects of metformin and HFD on serum insulin levels. Bars represent the means \pm SD from 3 independent experiments. ${ }^{* *} \mathrm{P}<0.01$ vs. Con; ${ }^{\#} \mathrm{P}<0.01$ vs. HFD. (C) HOMA-IR of experimental groups. Bars represent the means \pm SD from 3 independent experiments. ${ }^{* *} \mathrm{P}<0.01$ vs. Con; ${ }^{\# \#} \mathrm{P}<0.01$ vs. HFD. (D) Effects of metformin and HFD on urinary albumin excretion. Bars represent the means $\pm \mathrm{SD}$ from 3 independent experiments. ${ }^{*} \mathrm{P}<0.05$ vs. Con; " $\mathrm{P}<0.05$ vs. HFD. Con, control group fed standard diet; HFD, group fed high-fat diet; HFD + Met, group fed high-fat diet in conjunction with metformin; HOMA-IR, homeostasis model assessment of insulin resistance.

was significantly increased in the HFD-fed group compared with the control group. The HFD-induced increase in urinary albumin excretion was substantially reduced in the group administered metformin (Fig. 1D). These results suggest that the administration of metformin is effective in improving not only obesity-induced systemic metabolic derangement but also urinary albumin excretion.

Metformin decreases HFD-induced renal injury. The above data indicated that HFD increased urinary albumin secretion, suggesting that the kidneys were damaged. Therefore, we evaluated the morphological changes in the kidneys by PAS staining and electron microscopy. As compared with the control group, the mesangial area and Vglom were increased in the HFD-fed group. The administration of metformin ameliorated the HFD-induced glomerular changes (Fig. 2A-C). Electron microscopic analyses revealed segmental foot process effacement and tubular lipid droplets in the HFD-fed group. These ultrastructural changes were attenuated by the administration of metformin (Fig. 2D).

We then examiend HFD-induced glomerular fibrosis by immunofluorescence staining. The degree of the expression of glomerular fibronectin and type I and IV collagen was significantly higher in the HFD-fed group compared with the control group. The HFD-induced expression of glomerular fibrosis marker proteins was significantly reduced in the group administered metformin (Fig. 3). In addition, we evaluated HFD-induced glomerular podocyte changes. Glomerular synaptopodin expression was significantly decreased in the HFD-fed group compared witht the control group. However, synaptopodin expression was not decreased in the group administered metformin, showing similar levels to the control group (Fig. 4). These data suggest that metformin prevents HFD-induced glomerular changes, including fibrosis and podocyte injury.

Metformin decreases HFD-induced inflammatory response. Obesity is related to low-grade inflammatory process. We therefore assessed serum adipokine levels by ELISA. The serum levels of TNF- $\alpha$, IL-6, leptin, resistin, total PAI-1 and MCP-1 were significantly increased in the HFD-fed group compared with the control group (fed standard diet). The administration of metformin ameliorated the HFD-induced increase in serum adipokine levels. Serum adiponectin levels in the HFD-fed group were significantly lower than the levels in the control group. The HDF-induced decrease in adiponetin levels was not observed in the group administered metformin, showing similar levels to the control group (Fig. 5). Consistent with the degree of the expression of serum adipokine, renal F4/80-positive macrophage infiltration in the HFD-fed group was significantly increased in the tubulointerstitial areas. Macrophage infiltration in the group administered metformin was significantly lower than that in the HFD-fed group (Fig. 6). These results indicate that metformin modulates the expression of adipokines and reduces HFD-induced renal inflammation. 
A

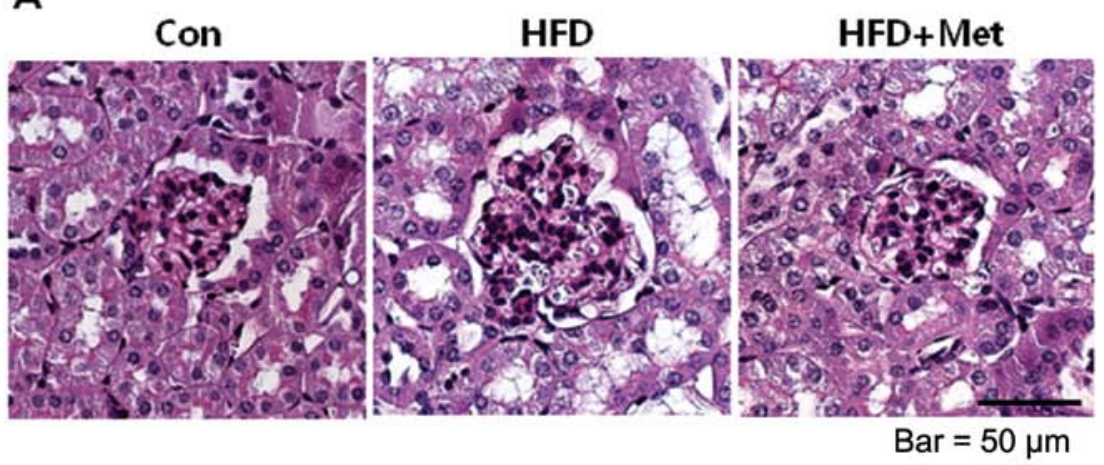

B
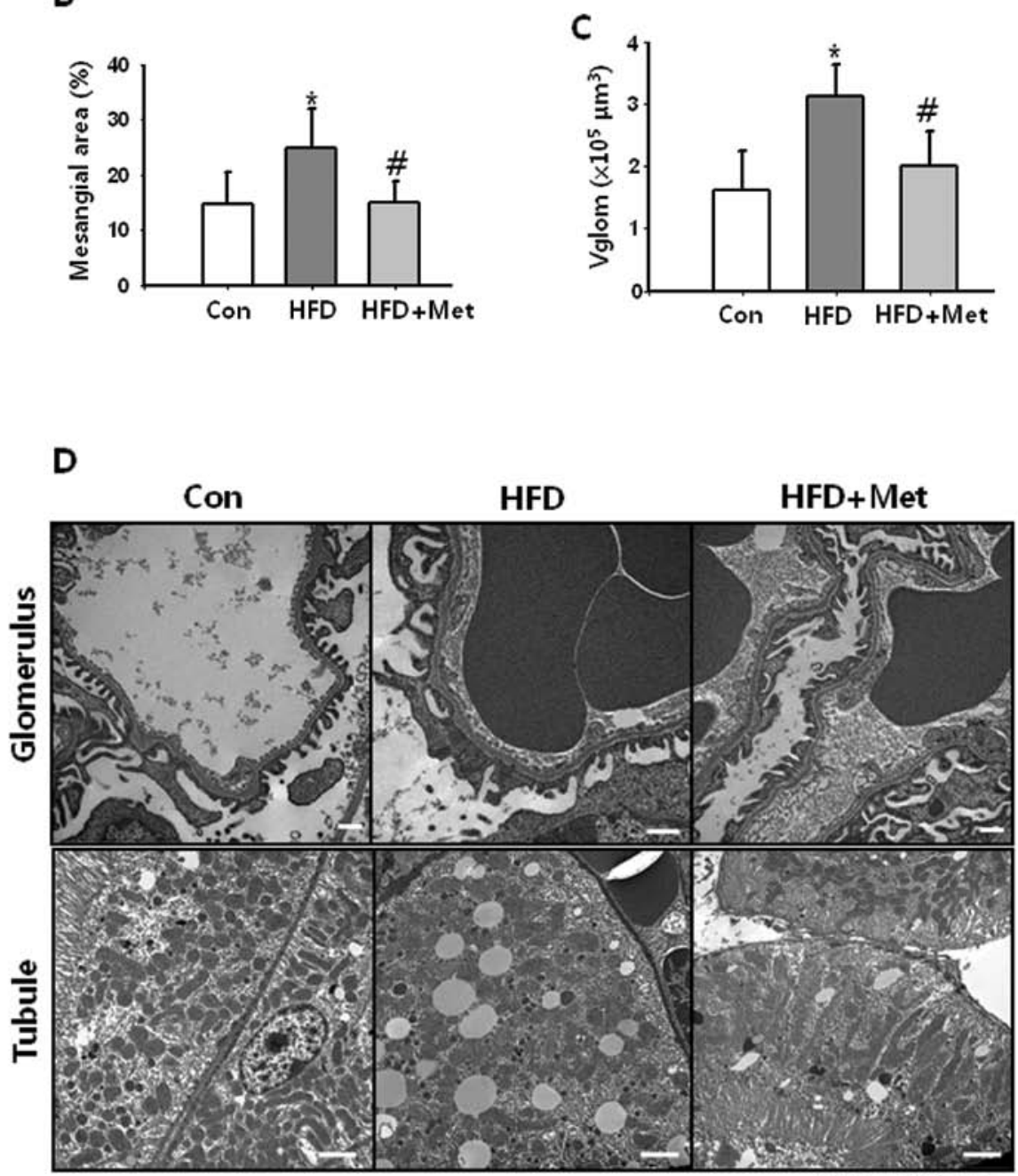

Bar $=500 \mathrm{~nm}$

Figure 2. Effects of metformin on renal histology in HFD-induced obese mice. (A) Representative histological sections of kidneys from mice fed standard diet, HFD and HFD in conjunction with metformin. (B) Mesangial areas of experimental animals. Bars represent the means \pm SD ( $n=12$ mice/each group) ${ }^{*} \mathrm{P}<0.05$ vs. Con; ${ }^{\#} \mathrm{P}<0.05$ vs. HFD. (C) Glomerular volume (Vglom) of experimental animals. Bars represent the means $\pm \mathrm{SD}$ ( $\mathrm{n}=12$ mice/each group). ${ }^{\text {P }}<0.05$ vs. Con; ${ }^{*} \mathrm{P}<0.05$ vs. HFD. (D) Representative electron miroscopic findings of glomeruli and tubules from experimental animals ( $\mathrm{n}=12 \mathrm{mice} / \mathrm{each}$ group). ${ }^{*} \mathrm{P}<0.05$ vs. Con; ${ }^{\#} \mathrm{P}<0.05$ vs. HFD. Con, control group fed standard diet; HFD, group fed high-fat diet; HFD + Met, group fed high-fat diet in conjunction with metformin.

Metformin increases renal fatty acid oxidation by the AMPK/ACC pathway. Metformin exerts beneficial effects against fatty liver disease by reducing hepatic lipid content through the coordination of the AMPK/ACC pathway (9).
Therefore, we assessed renal AMPK activity by western blot analysis. The phosphorylation of AMPK in the kidney tissues of the HFD-fed group was significantly decreased compared with the control group. However, the phosphoryla- 
A

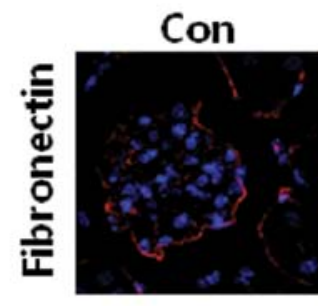

B

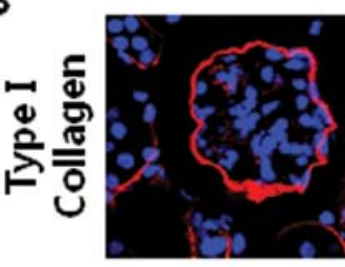

C

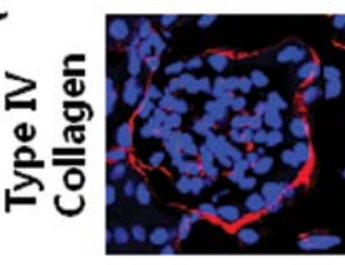

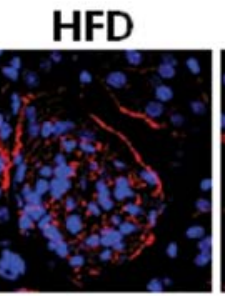
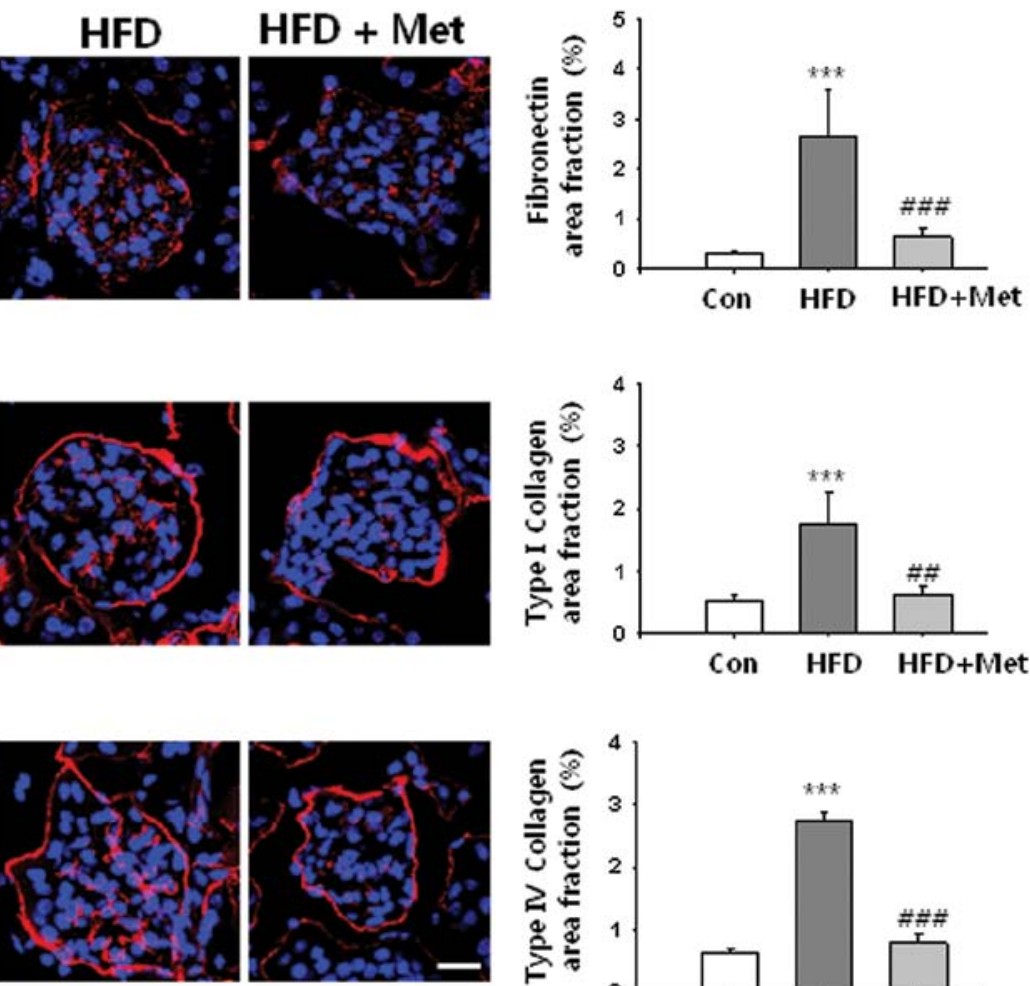

Bar $=20 \mu \mathrm{m}$

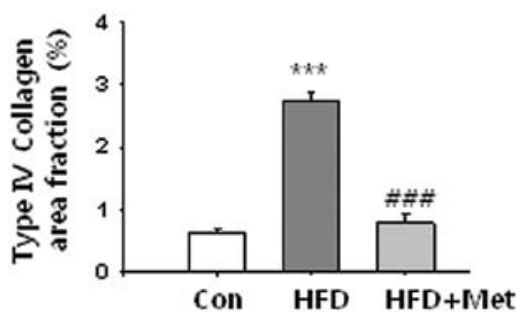

Figure 3. Effects of metformin on HFD-induced glomerular fibronectin, type collagen I and type IV collagen expression. Representative immunofluorescence staining for (A) fibronectin, (B) type I collagen and (C) type IV collagen from mice fed standard diet, HFD and HFD in conjunction with metformin and quantitative analysis of the area fraction (\%) of fibronectin, type I and IV collagen in each glomeruli. Bars represent the means $\pm \mathrm{SD}$ ( $\mathrm{n}=12$ for each experimental group) ${ }^{* * *} \mathrm{P}<0.001$ vs. Con; ${ }^{\# \# /} \mathrm{P}<0.001$ vs. HFD; ${ }^{\# *} \mathrm{P}<0.01$ vs. HFD. Con, control group fed standard diet; HFD, group fed high-fat diet; HFD + Met, group fed high-fat diet in conjunction with metformin.
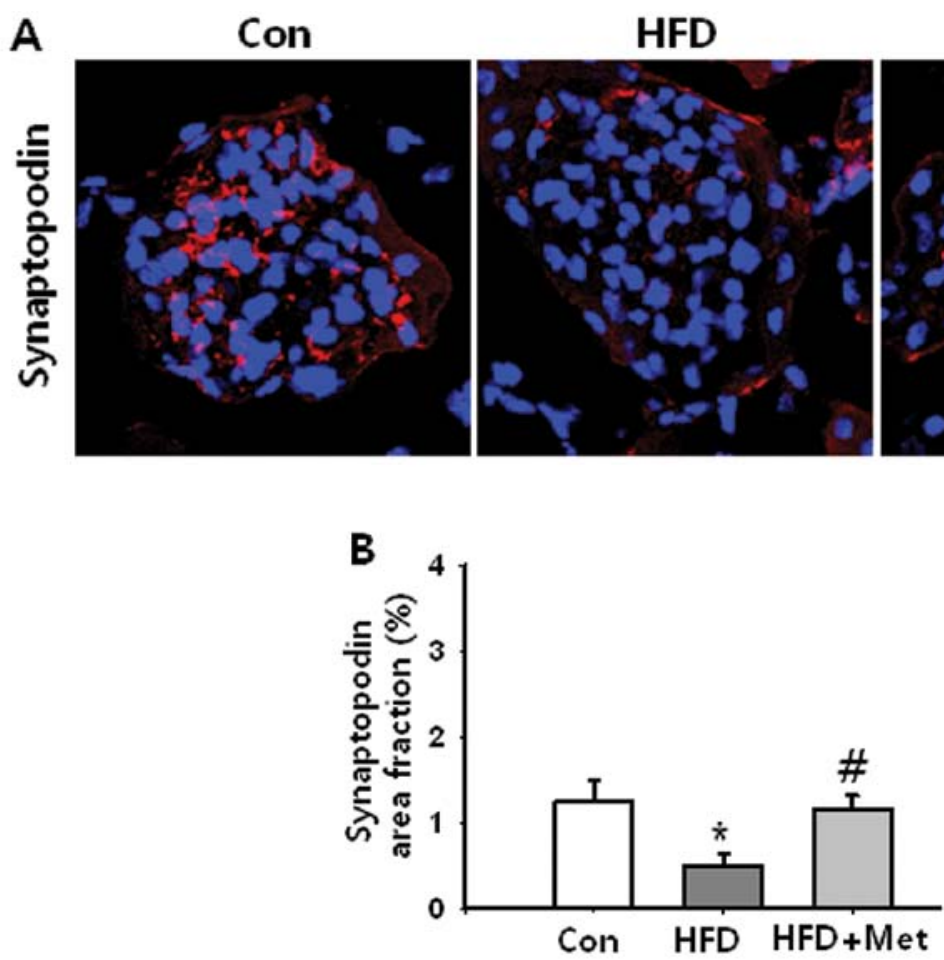

Figure 4. Effects of metformin on HFD-induced glomerular podocyte injury. (A) Representative sections of kidneys from mice fed standard diet, HFD and HFD in conjunction with metformin were stained by synaptopodin. (B) Quantitative analysis of area fraction (\%) of synaptopodin in each glomerulus. Bars represent the means $\pm \mathrm{SD}$ ( $\mathrm{n}=12$ for each experimental group) ${ }^{*} \mathrm{P}<0.05$ vs. Con; ${ }^{*} \mathrm{P}<0.05$ vs. HFD. Con, control group fed standard diet; HFD, group fed high-fat diet; HFD + Met, group fed high-fat diet in conjunction with metformin. 

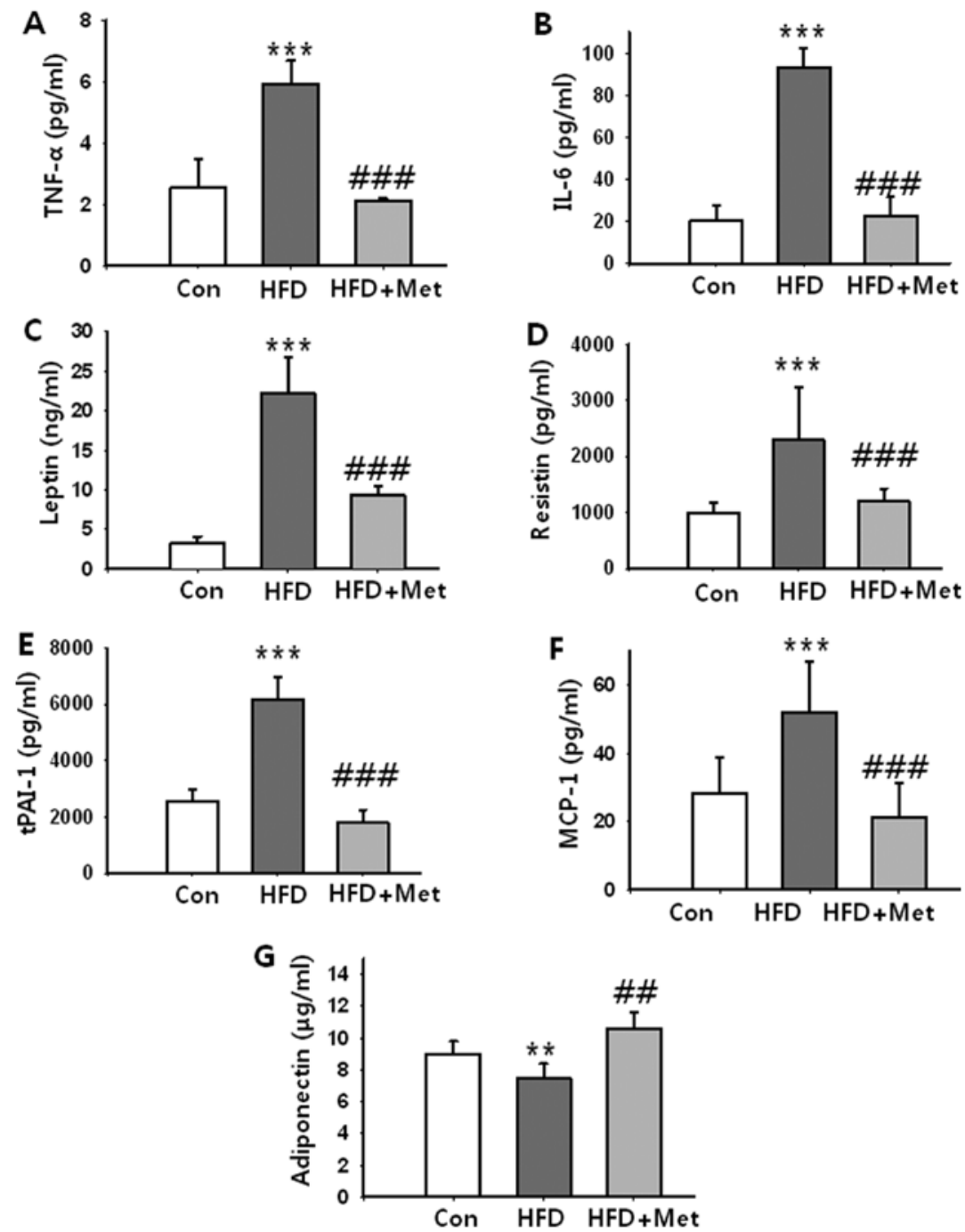

Figure 5. Effects of metformin on serum adipokine levels in HFD-induced obese mice. Serum levels of (A) TNF- $\alpha$, (B) IL-6, (C) leptin, (D) resistin, (E) tPAI-1, (F) MCP-1 and (G) adiponectin were measured by ELISA in mice fed standard diet, HFD and HFD in conjunction with metformin. Bars represent the means $\pm \mathrm{SD}$ ( $\mathrm{n}=12$ for each experimental group). ${ }^{* * *} \mathrm{P}<0.001$ vs. Con; ${ }^{* \# \#} \mathrm{P}<0.001$ vs. HFD. ${ }^{* *} \mathrm{P}<0.01$ vs. Con; ${ }^{\# \#} \mathrm{P}<0.01$ vs. HFD. Con, control group fed standard diet; HFD, group fed high-fat diet; HFD + Met, group fed high-fat diet in conjunction with metformin; TNF- $\alpha$, tumor necrosis factor- $\alpha$; IL-6, interleukin-6; tPAI-1, total plasminogen activator inhibitor-1; MCP-1, monocyte chemoattactant protein-1; ELISA, enzyme-linked immunosorbent assay.
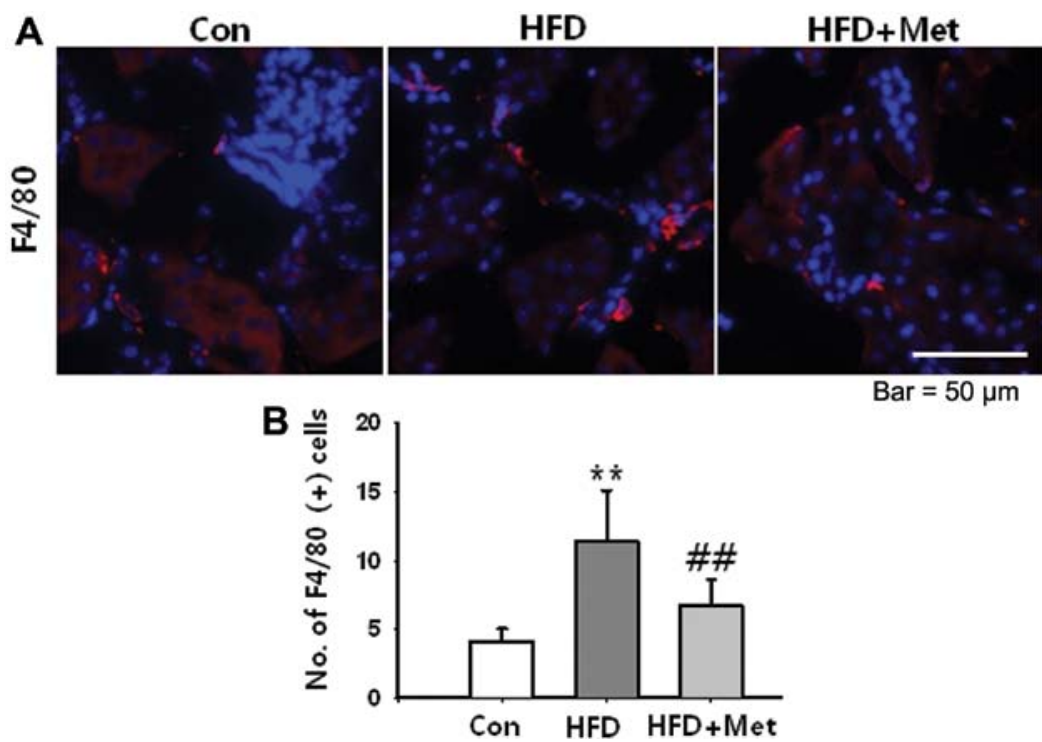

Figure 6. Effects of metformin on HFD-induced renal inflammatory cell infiltration. (A) Representative sections of kidneys from mice fed standard diet, HFD and HFD with metformin stained with F4/80. (B) Number of F4/80(+) cells/ $\mathrm{x} 400$ magnification. Bars represent the means $\pm \mathrm{SD}$ ( $\mathrm{n}=12$ for each experimental group). ${ }^{* *} \mathrm{P}<0.01$ vs. Con; ${ }^{\# \#} \mathrm{P}<0.01$ vs. HFD. Con, control group fed standard diet; HFD, group fed high-fat diet; HFD + Met, group fed high-fat diet in conjunction with metformin. 

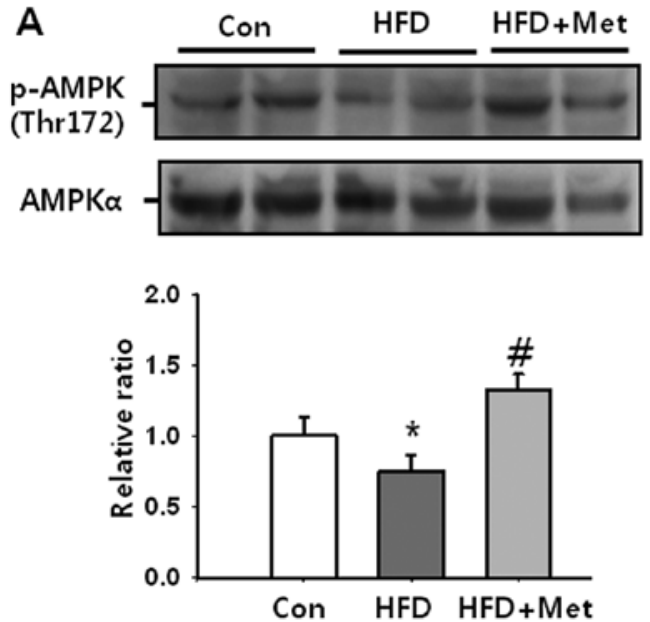
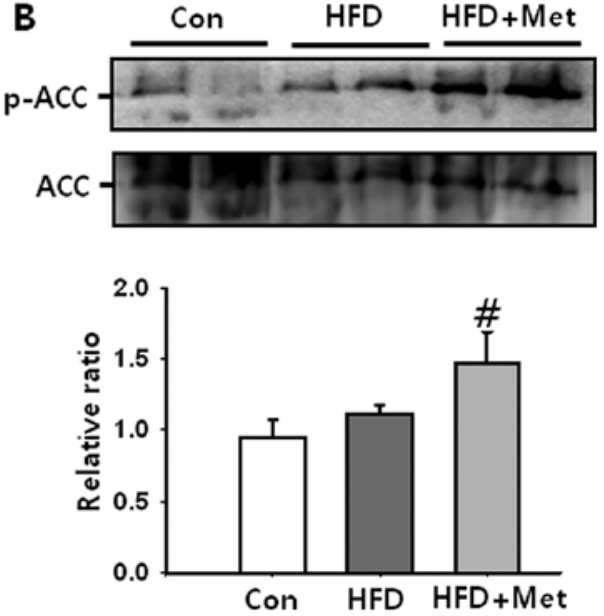

Figure 7. Effects of metformin on renal phospho-AMPK and phospho-ACC expression in HFD-induced obese mice. (A) Phospho-AMPK and (B) phospho-ACC expression in kidney tissues from mice fed standard diet, HFD and HFD with metformin was evaluated by western blot analysis. Densitometric analyses for phospho-AMPK and -ACC are presented as the relative ratio of each protein to total AMPK $\alpha$ and ACC, respectively. The relative ratio measured in the kidneys from the control mice is arbitrarily presented as 1 . Bars represent the means $\pm \mathrm{SD}$ from 3 independent experiments. ${ }^{*} \mathrm{P}<0.05$ vs. Con; ${ }^{*} \mathrm{P}<0.05$ vs. HFD. Con, control group fed standard diet; HFD, group fed high-fat diet; HFD + Met, group fed high-fat diet in conjunction with metformin; p-AMPK, phospho-AMPactivated protein kinase; AMPK, AMP-activated protein kinase; p-ACC, phospho-acetyl-CoA carboxylase; ACC, acetyl-CoA carboxylase.

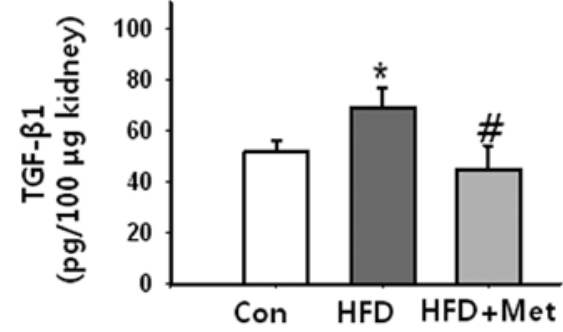

Figure 8. Effects of metformin on renal TGF- $\beta 1$ levels in HFD-induced obese mice. TGF- $\beta 1$ levels in kidney tissues from mice fed standard diet, HFD and HFD with metformin were evaluated by ELISA. The levels of renal TGF- $\beta 1$ normalized to $100 \mu \mathrm{g}$ of kidney. Bars represent the means $\pm \mathrm{SD}(\mathrm{n}=12$ for each experimental group). "P<0.05 vs. Con; ${ }^{\#} \mathrm{P}<0.05$ vs. HFD. Con, control group fed standard diet; HFD, group fed high-fat diet; HFD + Met, group fed high-fat diet in conjunction with metformin; TGF- $\beta 1$, trasnforming growth factor- $\beta 1$; ELISA, enzyme-linked immunosorbent assay.

tion of AMPK in the group administered metaformin was significantly higher than that in the HFD-fed group and the control group (Fig. 7A). In addition, a previous study reported that metformin inactivated ACC while stimulating fatty acid oxidation in rat primary hepatocytes (13). Therefore, we evaluated the phosphorylation of ACC. Compared with the control group, the phosphorylation of ACC was not increased in the kidney tissues of the HFD-fed group. However, the phosphorylation of ACC in the group administered metaformin was significantly higher compared with the HFD-fed group and the control group (Fig. 7B). These data suggest that metformin regulates fatty acid oxidation through the activation of AMPK and the deactivation of ACC.

Metformin decreases renal TGF- $\beta 1$ expression in HFD-induced renal injury. We assessed renal TGF- $\beta 1$ expression following HFD-induced renal injury by ELISA. Renal TGF- $\beta 1$ expression in the HFD-fed group was significantly increased compared with the control group. The HFD-induced increase in TGF- $\beta 1$ expression was substantially reduced in the group administered metformin (Fig. 8).

\section{Discussion}

In the present study, we demonstrate that HFD results in body and kidney weight gain, insulin resistance, increased adipokine expression and metabolic derangement. In addition, HFD-induced glomerular changes were evidenced by microalbuminuria and mesangial matrix expansion. Furthermore, mice fed HFD in conjunction with metformin showed a significant reduction in insulin resistance, inflammation and metabolic derangement, as well as in HFD-induced mesangial expansion and microalbuminuria. These findings suggest that metformin reduces HFD-induced renal injury.

The link between obesity and kidney injury has been explained by renal hyperfiltration. Dengel et al (22) reported that increased insulin resistance may predispose hypertensive subjects to renal injury by aggravating renal hemodynamic changes. Even during the early stages, HFD induces not only renal hemodynamic changes, including glomerular filtration and effective renal plasma flow, but also glomerular structural changes, such as the expansion of Bowman's capsule and mesangial matrix (23). Consistent with previous reports, we observed the HFD-induced increase in Vglom and mesangial matrix and metformin effectively ameliorated these glomerular structural changes.

In addition to renal hemodynamic changes, adipose tissuederived cytokines, adipokines, may also play an important role in the pathogenesis of obesity-related renal injury. Among these adipokines, leptin, known as anorexigenic hormone, stimulates endocapillary proliferation and mesangial cell collagen deposition via TGF- $\beta$ and its receptor expression in the glomerulus $(24,25)$. By contrast, adiponectin has anti-atherogenic and insulin-sensitizing properties $(26,27)$. Sharma et al (28) 
reported that adiponectin is a key regulator of albuminuria in a non-diabetic obese African-American population and plays a protective role in podocyte function through the AMPK pathway, modulating oxidative stress (28). Other adipokines, such as resistin, IL-6, PAI-1, MCP-1 and TNF- $\alpha$ exert proinflammatory properties and promote the increase in cell adhesion molecule expression in endothelial cells and vascular injury (26). In this context, in this study, HFD increased the levels of serum TNF- $\alpha$, IL-6, leptin, resistin, tPAI-1 and MCP-1 and decreased the levels of serum adiponectin. However, HFD in conjunction with metformin significantly reduced these increased levels of serum adipokines compared with the HFD-fed mice. The HFD-induced increase in macrophage infiltration into the kidneys was decreased following the administration of metformin. Taken together, these results suggest that metformin decreases systemic and local inflammatory conditions induced by obesity, leading to an improvement of obesity-related renal injury.

Obesity is related to an excess energy fuel state, which results in the suppression of AMPK activity. The decreased AMPK activity leads to cellular hypertrophy and mesangial expansion with matrix accumulation in the kidneys (29). In this study, we demonstrated that HFD decreased renal AMPK activity. Metformin, an AMPK activator, reversed the HFD-induced decrease in renal AMPK activity. In terms of renal function, metformin markedly decreased urinary albumin excretion induced by HFD. Our results revealed that metformin improved HFD-induced insulin resistance and modulated adipokine expression. Thus, the renoprotective effects of metformin are partially responsible for the improvement of AMPK activity, as well as the improvement of inflammation and insulin resistance.

Additionally, podocytopathy is another hallmark of obesityrelated renal injury. From the classic point of view, albuminuria results in changes in the size- and charge-selective glomerular filtration barrier (30). Synaptopodin, a proline-rich actinassociated protein, is expressed in podocyte foot processes and its role is maintaining podocyte foot process structure and preserving glomerular barrier function (31). The overexpression of TGF- $\beta 1$ in the rat glomeruli induces proteinuria and decreases the expression of nephrin and synaptopodin in immortalized podocytes (32). Consistent with previous observations, our ultrastructural study indicated segmental foot process effacement in the HFD-fed group. The expression of synaptopodin was decreased in the glomerulus in the HFD-fed group. Renal TGF- $\beta 1$ expression was increased with HFD. The administration of metformin improved HFD-induced foot process effacement and the expression of glomerular synaptopodin. These results indicate that metformin exerts a protective effect on HFD-induced podocyte injury by regulating the expression of renal AMPK and TGF- $\beta 1$.

Another mechanism of obesity-related renal injury is related to the abnormal accumulation of lipids in the glomeruli and renal tubules. The accumulation of excess renal lipids in diabetic or obese animals correlates with markers of renal dysfunction, such as microalbuminuria, mesangial matrix accumulation and tubulointerstitial inflammation, while genetic, pharmacological or dietary intervention improves renal lipid accumulation, as well as histological or functional injury (33). Our electron microscopic examination revealed that HFD increased lipid accumulation in tubular epithelial cells. Pharmacological intervention by metformin decreased renal lipid accumulation, as well as dyslipidemia.

Studies have demonstrated that metformin decreases basal hepatic glucose output by the inhibition of gluconeogenesis and insulin-mediated glucose utilization in peripheral tissues, such as muscle and liver tissue $(9,34)$. In addition, metformin exerts a beneficial effect on lipid metabolism, reversing hepatic steatosis (13). In this study, we demonstrated that the administration of metformin reversed the HFD-induced decrease in renal AMPK activity. Moreover, metformin increased the phosphorylation of ACC, a rate-controlling enzyme for fatty acid metabolism, which was decreased by HFD. Therefore, the overall effect of increased AMPK activity induced by metformin in this study may be related to the stimulation of fatty acid oxidation.

In conclusion, our study demonstrates that metformin attenuates HFD-induced glomerular injury in mice by regulating HFD-induced metabolic derangement, renal fatty acid metabolism and serum adipokine expression. Our results suggest that the mechanisms behind the protective effects of metformin involve the regulation of obesity-induced systemic inflammation, insulin resistance and the renal AMPK/ACC pathway. The clinical application of metformin to obese or early diabetic patients may help prevent and/or treat obesity- or diabetes-related kidney disease.

\section{Acknowledgements}

The present study was supported by the National Research Foundation of Korea (NRF) funded by the Korean government (2012R1A1A4A01010065 and 2012-0009322, to K.P.K).

\section{References}

1. Bagby SP: Obesity-initiated metabolic syndrome and the kidney: a recipe for chronic kidney disease? J Am Soc Nephrol 15: 2775-2791, 2004

2. Chen J, Muntner P, Hamm LL, et al: Insulin resistance and risk of chronic kidney disease in nondiabetic US adults. J Am Soc Nephrol 14: 469-477, 2003.

3. Chen J, Muntner P, Hamm LL, et al: The metabolic syndrome and chronic kidney disease in U.S. adults. Ann Intern Med 140: 167-174, 2004.

4. Agrawal V, Shah A, Rice C, Franklin BA and McCullough PA: Impact of treating the metabolic syndrome on chronic kidney disease. Nat Rev Nephrol 5: 520-528, 2009.

5. Maury E and Brichard SM: Adipokine dysregulation, adipose tissue inflammation and metabolic syndrome. Mol Cell Endocrinol 314: 1-16, 2010.

6. Tang J, Yan $\mathrm{H}$ and Zhuang S: Inflammation and oxidative stress in obesity-related glomerulopathy. Int J Nephrol 2012: 608397, 2012.

7. Towler MC and Hardie DG: AMP-activated protein kinase in metabolic control and insulin signaling. Circ Res 100: 328-341, 2007.

8. Nathan DM, Buse JB, Davidson MB, et al: Medical management of hyperglycemia in type 2 diabetes: a consensus algorithm for the initiation and adjustment of therapy: a consensus statement of the American Diabetes Association and the European Association for the Study of Diabetes. Diabetes Care 32: 193-203, 2009.

9. Viollet B, Guigas B, Sanz Garcia N, Leclerc J, Foretz M and Andreelli F: Cellular and molecular mechanisms of metformin: an overview. Clin Sci (Lond) 122: 253-270, 2012.

10. El-Mir MY, Nogueira V, Fontaine E, Avéret N, Rigoulet M and Leverve X: Dimethylbiguanide inhibits cell respiration via an indirect effect targeted on the respiratory chain complex I. J Biol Chem 275: 223-228, 2000. 
11. Owen MR, Doran E and Halestrap AP: Evidence that metformin exerts its anti-diabetic effects through inhibition of complex 1 of the mitochondrial respiratory chain. Biochem J 348: 607-614, 2000.

12. Salminen A, Hyttinen JM and Kaarniranta K: AMP-activated protein kinase inhibits NF- $\kappa$ B signaling and inflammation: impact on healthspan and lifespan. J Mol Med (Berl) 89: 667-676, 2011.

13. Zhou G, Myers R, Li Y, et al: Role of AMP-activated protein kinase in mechanism of metformin action. J Clin Invest 108: 1167-1174, 2001.

14. Viollet B, Guigas B, Leclerc J, et al: AMP-activated protein kinase in the regulation of hepatic energy metabolism: from physiology to therapeutic perspectives. Acta Physiol (Oxf) 196 81-98, 2009.

15. Abu-Elheiga L, Matzuk MM, Abo-Hashema KA and Wakil SJ: Continuous fatty acid oxidation and reduced fat storage in mice lacking acetyl-CoA carboxylase 2. Science 291: 2613-2616, 2001.

16. Decleves AE, Mathew AV, Cunard R and Sharma K: AMPK mediates the initiation of kidney disease induced by a high-fat diet. J Am Soc Nephrol 22: 1846-1855, 2011.

17. Kita Y, Takamura T, Misu H, et al: Metformin prevents and reverses inflammation in a non-diabetic mouse model of nonalcoholic steatohepatitis. PLoS One 7: e43056, 2012.

18. Lane PH, Steffes MW and Mauer SM: Estimation of glomerular volume: a comparison of four methods. Kidney Int 41: 1085-1089, 1992.

19. Kim DH, Jung YJ, Lee AS, et al: COMP-angiopoietin-1 decreases lipopolysaccharide-induced acute kidney injury. Kidney Int 76 1180-1191, 2009.

20. Kang KP, Kim DH, Jung YJ, et al: Alpha-lipoic acid attenuates cisplatin-induced acute kidney injury in mice by suppressing renal inflammation. Nephrol Dial Transplant 24: 3012-3020, 2009.

21. Kang KP, Park SK, Kim DH, et al: Luteolin ameliorates cisplatin-induced acute kidney injury in mice by regulation of p53-dependent renal tubular apoptosis. Nephrol Dial Transplant 26: 814-822, 2011.

22. Dengel DR, Goldberg AP, Mayuga RS, Kairis GM and Weir MR: Insulin resistance, elevated glomerular filtration fraction, and renal injury. Hypertension 28: 127-132, 1996.
23. Henegar JR, Bigler SA, Henegar LK, Tyagi SC and Hall JE: Functional and structural changes in the kidney in the early stages of obesity. J Am Soc Nephrol 12: 1211-1217, 2001.

24. Wolf G, Hamann A, Han DC, et al: Leptin stimulates proliferation and TGF-beta expression in renal glomerular endothelial cells: potential role in glomerulosclerosis [see comments]. Kidney Int 56: 860-872, 1999.

25. Han DC, Isono M, Chen S, et al: Leptin stimulates type I collagen production in $\mathrm{db} / \mathrm{db}$ mesangial cells: glucose uptake and TGF-beta type II receptor expression. Kidney Int 59: 1315-1323, 2001.

26. Chudek J, Adamczak M, Nieszporek T and Wiecek A: The adipose tissue as an endocrine organ - a nephrologists' perspective. Contrib Nephrol 151: 70-90, 2006.

27. Diez JJ and Iglesias P: The role of the novel adipocyte-derived hormone adiponectin in human disease. Eur J Endocrinol 148: 293-300, 2003.

28. Sharma K, Ramachandrarao S, Qiu G, et al: Adiponectin regulates albuminuria and podocyte function in mice. J Clin Invest 118: 1645-1656, 2008.

29. Ix JH and Sharma K: Mechanisms linking obesity, chronic kidney disease, and fatty liver disease: the roles of fetuin-A, adiponectin, and AMPK. J Am Soc Nephrol 21: 406-412, 2010.

30. Deen WM: What determines glomerular capillary permeability? J Clin Invest 114: 1412-1414, 2004.

31. Mundel P, Heid HW, Mundel TM, Kruger M, Reiser J and Kriz W: Synaptopodin: an actin-associated protein in telencephalic dendrites and renal podocytes. J Cell Biol 139: 193-204, 1997.

32. Ghayur A, Liu L, Kolb M, et al: Adenovirus-mediated gene transfer of TGF-beta1 to the renal glomeruli leads to proteinuria. Am J Pathol 180: 940-951, 2012.

33. Bobulescu IA: Renal lipid metabolism and lipotoxicity. Curr Opin Nephrol Hypertens 19: 393-402, 2010.

34. Bailey CJ and Turner RC: Metformin. N Engl J Med 334: 574-579, 1996. 